\title{
Analysis of Superconductivity in d-p Model on Basis of Perturbation Theory
}

\author{
Sotaro SAsAKI, Hiroaki IKEDA and Kosaku YAmadA \\ Department of Physics, Kyoto University, Sakyo-ku, Kyoto 606-8502
}

(Received September 12, 2018)

\begin{abstract}
We investigate the mass enhancement factor and the superconducting transition temperature in the d-p model for the high- $T_{\mathrm{c}}$ cuprates. We solve the Éliashberg equation using the thirdorder perturbation theory with respect to the on-site Coulomb repulsion $U$. We find that when the energy difference between the d-level and p-level is large, the mass enhancement factor becomes large and $T_{\mathrm{c}}$ tends to be suppressed owing to the difference of the density of state for d-electron at the Fermi level. From another viewpoint, when the energy difference is large, the d-hole number approaches unity and the electron correlation becomes strong and enhances the effective mass. This behavior for the electron number is the same as that for the f-electron number in the heavy fermion systems. The mass enhancement factor plays an essential role in understanding the difference in $T_{\mathrm{c}}$ between the LSCO and YBCO systems.
\end{abstract}

KEYWORDS: $\mathrm{d}-\mathrm{p}$ model, high- $T_{\mathrm{c}}$ cuprate, mass enhancement factor, transition temperature, third-order perturbation theory

Currently, superconductivity in high- $T_{\mathrm{c}}$ cuprates is being intensively investigated. For example, from the point of view of strong coupling, the $t$ - $J$ model has been investigated. ${ }^{1}$ Also, Hubbard model has been investigated using the variational Monte Carlo method ${ }^{2}$ and the quantum Monte Carlo method. ${ }^{3}$ On the other hand, from the point of view of weak coupling, the Hubbard model has been investigated using the fluctuation-exchange approximation and the perturbation theory. In the latter case, the nature of superconductivity in the high- $T_{\mathrm{c}}$ cuprates has almost been clarified on the basis of the nearly antiferromagnetic Fermi-liquid theory. ${ }^{4,5}$ However, we have not yet explained the observed differences between the high- $T_{\mathrm{c}}$ systems, $\mathrm{YBa}_{2} \mathrm{Cu}_{3} \mathrm{O}_{7-\delta}(\mathrm{YBCO})$ and $\mathrm{La}_{2-x} \mathrm{Sr}_{x} \mathrm{CuO}_{4}$ (LSCO) and so on, in particular, the principal reason why the transition temperature $T_{\mathrm{c}}$ observed in LSCO is relatively low. The nuclear quadrupole resonance (NQR) experiment by Zheng et al. shows that when the ratio of the d-hole number $\left(n_{d}\right)$ to the phole number $\left(n_{p}\right), n_{d} / 2 n_{p}$, is large, $T_{\mathrm{c}}$ is suppressed. ${ }^{6}$ Actually, LSCO has a large ratio, as compared with YBCO. Also, in the specific heat experiment, the $\gamma$-value per mole in LSCO is approximately as large as that in YBCO. ${ }^{7,8}$ Considering that $\mathrm{YBCO}$ has two $\mathrm{CuO}_{2}$ layers and three $\mathrm{Cu}$ atoms in the unit, we find that the $\gamma$-value per layer in LSCO is large. The effect of the strong mass enhancement can be also seen in the nuclear magnetic resonance (NMR) relaxation rate. From the systematic study of $\left(T_{1} T\right)^{-1}$, we can see that the effective Fermi energy in LSCO is rather smaller than that in YBCO.${ }^{9}$ Thus, the strong electron correlation leads to a large mass enhancement, and then the $T_{\mathrm{c}}$ in LSCO is reduced. This proposal is very important to clarify a relation to the other materials, such as the heavy fermion systems, and to promote further progress on the unified picture in the strongly correlated systems. In this study, using the third-order perturbation theory, we investigate the mass enhancement factor and $T_{\mathrm{c}}$ in the d-p model for high- $T_{\mathrm{c}}$ cuprates, and clarify the difference in $T_{\mathrm{c}}$ be-

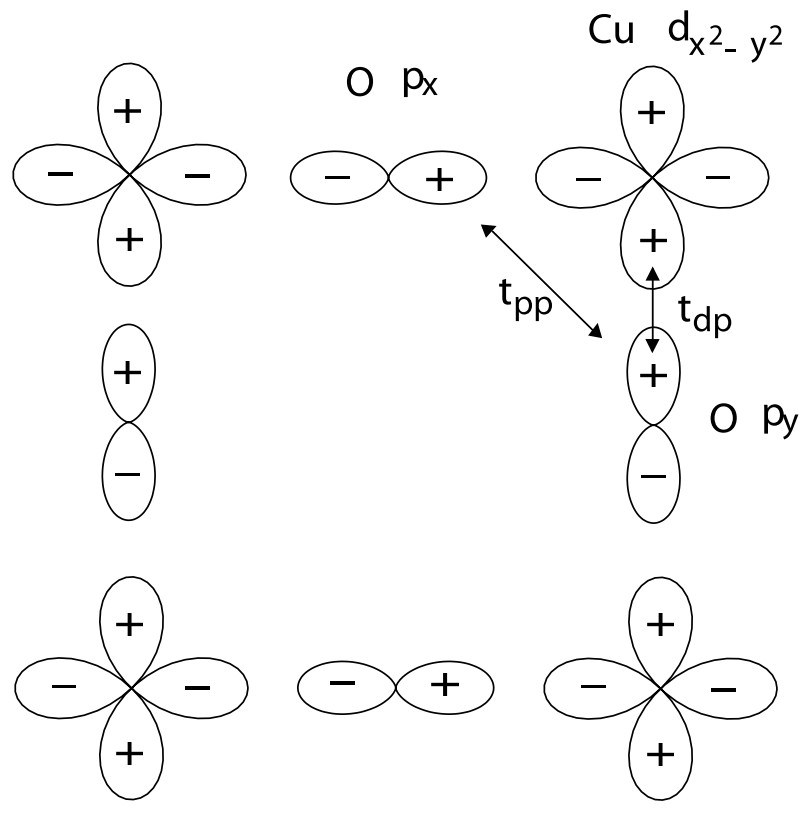

Fig. 1. Lattice structure of d-p model. $t_{\mathrm{dp}}$ and $t_{\mathrm{pp}}$ are the hopping integrals.

tween LSCO and YBCO. Husimi discussed it in the same framework and concluded that it originates from the difference in the effective on-site Coulomb repulsion $U .{ }^{10}$ We would like to stress here that the mass enhancement factor plays an essential role.

Since the $\mathrm{CuO}_{2}$ plane is essential for superconductivity in high- $T_{\mathrm{c}}$ cuprates, we can consider the lattice structure with only the $\mathrm{CuO}_{2}$ network shown in Fig. 1. This structure has the $d_{x^{2}-y^{2}}$ orbitals on $\mathrm{Cu}$ sites and the $p_{x}$ and $p_{y}$ orbitals on $\mathrm{O}$ sites in the primitive cell. For simplicity, we consider only the hopping integrals $t_{d p}$ and $t_{p p}$ shown in Fig. 1. This is the so-called d-p model. In this study, we investigate in detail the nature of superconductivity 


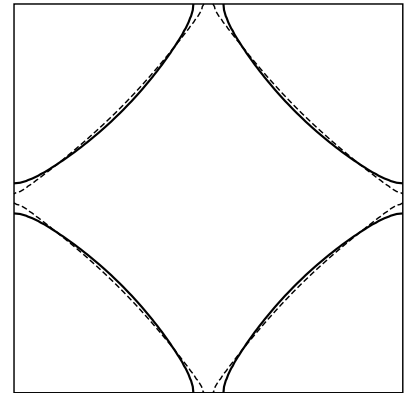

Fig. 2. The unbroken and broken lines are the Fermi surfaces in the case of $T=0.01, t_{\mathrm{pp}}=0.30$ and $n=4.90$ for $\varepsilon_{d}-\varepsilon_{p}=1.0$ and $\varepsilon_{d}-\varepsilon_{p}=3.0$, respectively.

in the d-p model. The model Hamiltonian is written as

$$
H=H_{0}+H_{\text {int }},
$$

where,

$H_{0}=\left(\begin{array}{lll}d_{k \sigma}^{\dagger} & p_{k \sigma}^{x \dagger} & p_{k \sigma}^{y \dagger}\end{array}\right)\left(\begin{array}{lll}\varepsilon_{d} & \xi_{k}^{x} & \xi_{k}^{y} \\ \xi_{k}^{x} & \varepsilon_{p} & \xi_{k}^{p} \\ \xi_{k}^{y} & \xi_{k}^{p} & \varepsilon_{p}\end{array}\right)\left(\begin{array}{c}d_{k \sigma} \\ p_{k \sigma}^{x} \\ p_{k \sigma}^{y}\end{array}\right)$.

Here, $\varepsilon_{d}$ and $\varepsilon_{p}$ include the chemical potential $\mu$. The essential parameter is the level splitting $\varepsilon_{d}-\varepsilon_{p}$. The offdiagonal parts are given by

$$
\begin{aligned}
& \xi_{k}^{i}=-2 t_{d p} \sin \frac{k_{i}}{2},(i=x, y), \\
& \xi_{k}^{p}=4 t_{p p} \sin \frac{k_{x}}{2} \sin \frac{k_{y}}{2} .
\end{aligned}
$$

The second term denotes the on-site Coulomb repulsion between the d-electrons,

$$
H_{\mathrm{int}}=\frac{U}{N} \sum_{k} \sum_{q \neq 0} d_{k+q \uparrow}^{\dagger} d_{k^{\prime}-q \downarrow}^{\dagger} d_{k^{\prime} \downarrow} d_{k \uparrow} .
$$

We set $t_{d p}=1.0$ as an energy unit, and we also fix $t_{p p}=0.30$ and $n=4.90$ to reproduce the Fermi surfaces. In Fig. 2, we show the Fermi surfaces for $\varepsilon_{d}-\varepsilon_{p}=1.0$ and $\varepsilon_{d}-\varepsilon_{p}=3.0$ in the case of $T=0.01$. We see that the Fermi surfaces coincide with those of the high- $T_{\mathrm{c}}$ cuprates, YBCO and LSCO, respectively. In this model, the electron number $n<5.0$ represents the hole-doped case, and $n>5.0$ represents the electron-doped case. We fix $T=0.01$ except when we discuss $T_{\mathrm{c}}$. Figs. 3 and 4 show the band structures and the density of states for d-electrons $\rho_{d}(\varepsilon)$ for $\varepsilon_{d}-\varepsilon_{p}=1.0$ and $\varepsilon_{d}-\varepsilon_{p}=3.0$, respectively. We see that when $\varepsilon_{d}-\varepsilon_{p}$ is large, $\rho_{d}(\varepsilon=0)$ at the Fermi level is large.

The bare Green's function $\hat{G}^{(0)}(k)$ is written as

$$
\hat{G}^{(0)}(k)=\left(i \omega_{n} \hat{1}-\hat{H}_{0}\right)^{-1} .
$$

We define the matrix elements of $\hat{G}^{(0)}(k)$ as

$$
\hat{G}^{(0)}(k)=\left(\begin{array}{ccc}
G_{\mathrm{dd}}^{(0)}(k) & G_{\mathrm{dp}_{\mathrm{x}}}^{(0)}(k) & G_{\mathrm{dp}_{\mathrm{y}}}^{(0)}(k) \\
G_{\mathrm{pxd}_{\mathrm{d}}}^{(0)}(k) & G_{\mathrm{p}_{x} \mathrm{p}_{\mathrm{x}}}^{(0)}(k) & G_{\mathrm{p}_{\mathrm{x}} \mathrm{p}_{\mathrm{y}}}^{(0)}(k) \\
G_{\mathrm{p}_{\mathrm{y}} \mathrm{d}}^{(0)}(k) & G_{\mathrm{p}_{\mathrm{y}} \mathrm{p}_{\mathrm{x}}}^{(0)}(k) & G_{\mathrm{p}_{\mathrm{y}} \mathrm{p}_{\mathrm{y}}}^{(0)}(k)
\end{array}\right) .
$$

We need $G_{\mathrm{dd}}^{(0)}(k)$ to only describe the normal self-energy

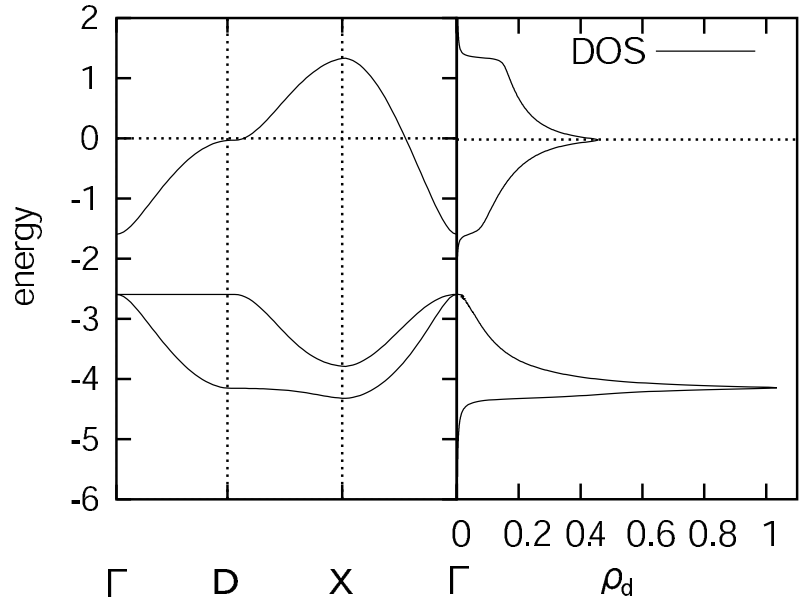

Fig. 3. Band structure and density of states for d-electron $\rho_{d}(\varepsilon)$ in case of $\varepsilon_{d}-\varepsilon_{p}=1.0$.

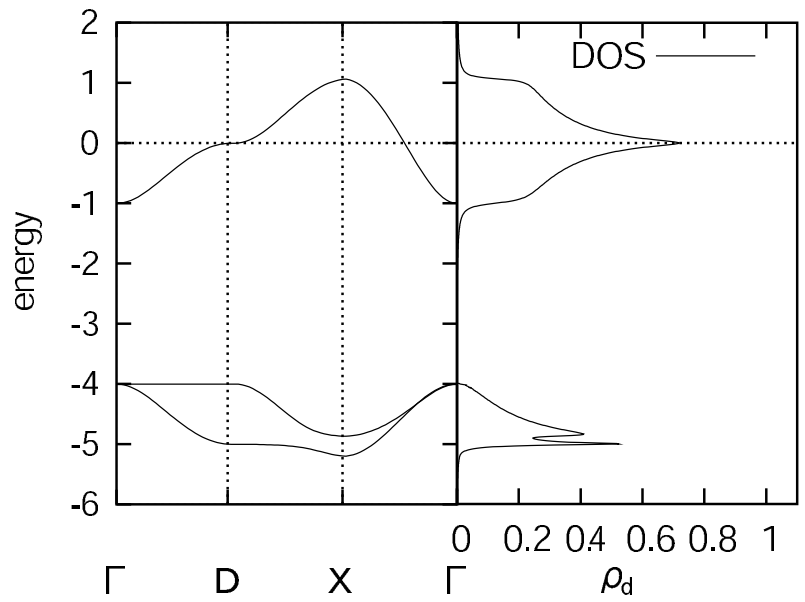

Fig. 4. Band structure and density of states for d-electron $\rho_{d}(\varepsilon)$ in case of $\varepsilon_{d}-\varepsilon_{p}=3.0$.

$\hat{\Sigma}(k)$ and the anomalous self-energy $\Delta(k)$, since the interaction exists only between the d-electrons.

$$
\hat{\Sigma}(k)=\left(\begin{array}{ccc}
\Sigma_{\mathrm{dd}}(k) & 0 & 0 \\
0 & 0 & 0 \\
0 & 0 & 0
\end{array}\right) .
$$

The Green's function is given by

$$
\hat{G}(k)=\left(i \omega_{n} \hat{1}-\hat{H}_{0}-\hat{\Sigma}(k)\right)^{-1} .
$$

In this study, the chemical potential $\mu$ is determined so as to fix the total electron number, $n$,

$$
n=2 \frac{T}{N} \sum_{k} \operatorname{Tr} \hat{G}_{0}(k)=2 \frac{T}{N} \sum_{k} \operatorname{Tr} \hat{G}(k) .
$$

We apply the third-order perturbation theory with respect to $U$. The normal self-energy is given by

$$
\begin{aligned}
\Sigma_{\mathrm{dd}}(k) & =\frac{T}{N} \sum_{k^{\prime}}\left[U^{2} \chi_{0}\left(k-k^{\prime}\right) G_{\mathrm{dd}}^{(0)}\left(k^{\prime}\right)\right. \\
& \left.+U^{3}\left(\chi_{0}^{2}\left(k-k^{\prime}\right)+\phi_{0}^{2}\left(k+k^{\prime}\right)\right) G_{\mathrm{dd}}^{(0)}\left(k^{\prime}\right)\right] .
\end{aligned}
$$




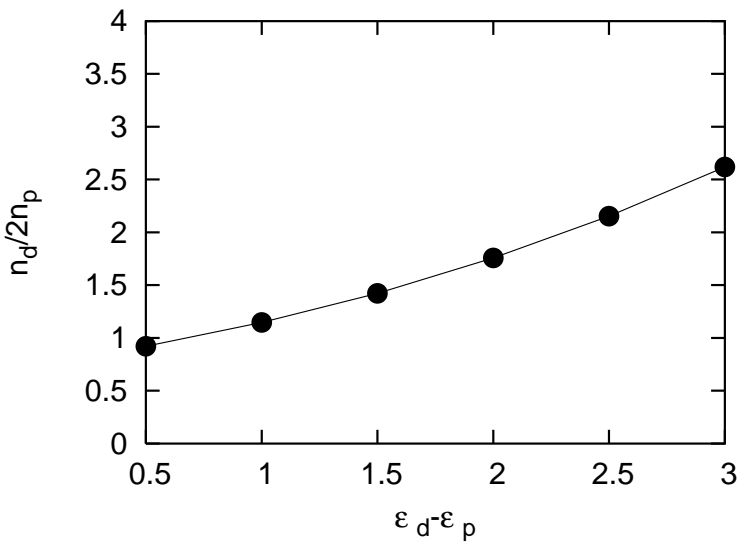

Fig. 5. $\varepsilon_{d}-\varepsilon_{p}$ dependence of $n_{d} / 2 n_{p}$.

Since the first-order term is constant, we can include the first-order term in $\varepsilon_{d}-\varepsilon_{p}$. Here,

$$
\begin{aligned}
& \chi_{0}(q)=-\frac{T}{N} \sum_{k} G_{\mathrm{dd}}^{(0)}(k) G_{\mathrm{dd}}^{(0)}(q+k), \\
& \phi_{0}(q)=-\frac{T}{N} \sum_{k} G_{\mathrm{dd}}^{(0)}(k) G_{\mathrm{dd}}^{(0)}(q-k) .
\end{aligned}
$$

We also expand the effective pairing interaction up to the third-order terms with respect to $U$.

For the spin-singlet state, the effective pairing interaction is given by

$$
V\left(k ; k^{\prime}\right)=V_{\mathrm{RPA}}\left(k ; k^{\prime}\right)+V_{\mathrm{Vertex}}\left(k ; k^{\prime}\right),
$$

where

$$
V_{\mathrm{RPA}}\left(k ; k^{\prime}\right)=U+U^{2} \chi_{0}\left(k-k^{\prime}\right)+2 U^{3} \chi_{0}^{2}\left(k-k^{\prime}\right),
$$

and

$$
\begin{aligned}
& V_{\text {Vertex }}\left(k ; k^{\prime}\right)=2(T / N) \operatorname{Re}\left[\sum_{k_{1}} G_{\mathrm{dd}}^{(0)}\left(k_{1}\right)\right. \\
& \left.\times\left(\chi_{0}\left(k+k_{1}\right)-\phi_{0}\left(k+k_{1}\right)\right) G_{\mathrm{dd}}^{(0)}\left(k+k_{1}-k^{\prime}\right) U^{3}\right] .
\end{aligned}
$$

Here, $V_{\mathrm{RPA}}\left(k, k^{\prime}\right)$ is called the RPA terms and $V_{\text {Vertex }}\left(k, k^{\prime}\right)$ is called the vertex corrections. Near the transition point, the anomalous self-energy $\Delta(k)$ satisfies the linearized Éliashberg equation,

$$
\lambda_{\max } \Delta(k)=-\frac{T}{N} \sum_{k^{\prime}} V\left(k ; k^{\prime}\right)\left|G_{\mathrm{dd}}\left(k^{\prime}\right)\right|^{2} \Delta\left(k^{\prime}\right),
$$

where $\lambda_{\max }$ is the largest positive eigenvalue. In this equation, the temperature with $\lambda_{\max }=1$ corresponds to $T_{\mathrm{c}}$. The symmetry of the superconductivity obtained here is the spin-singlet $d_{x^{2}-y^{2}}$ wave.

We take $64 \times 64 \boldsymbol{k}$-meshes for the first Brillouin zone and 2048 Matsubara frequencies in the numerical calculation. First, we show the physical quantities in the unperturbed state. In Fig. 5, we show the $\varepsilon_{d}-\varepsilon_{p}$ dependence of $n_{d} / 2 n_{p}$. When $\varepsilon_{d}-\varepsilon_{p}$ is large, $n_{d} / 2 n_{p}$ is large. Therefore, in the d-p model, the difference between LSCO and YBCO is represented as the difference of $\varepsilon_{d}-\varepsilon_{p}$. That is

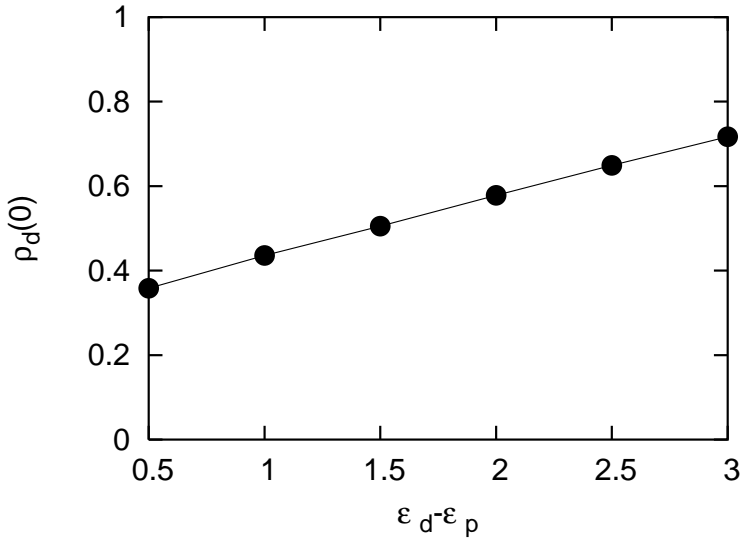

Fig. 6. $\varepsilon_{d}-\varepsilon_{p}$ dependence of $\rho_{d}(0)$.

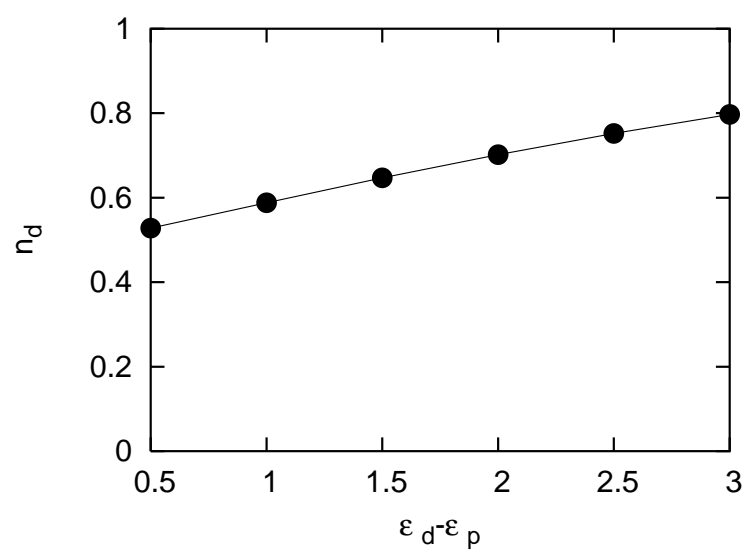

Fig. 7. $\varepsilon_{d}-\varepsilon_{p}$ dependence of $n_{d}$.

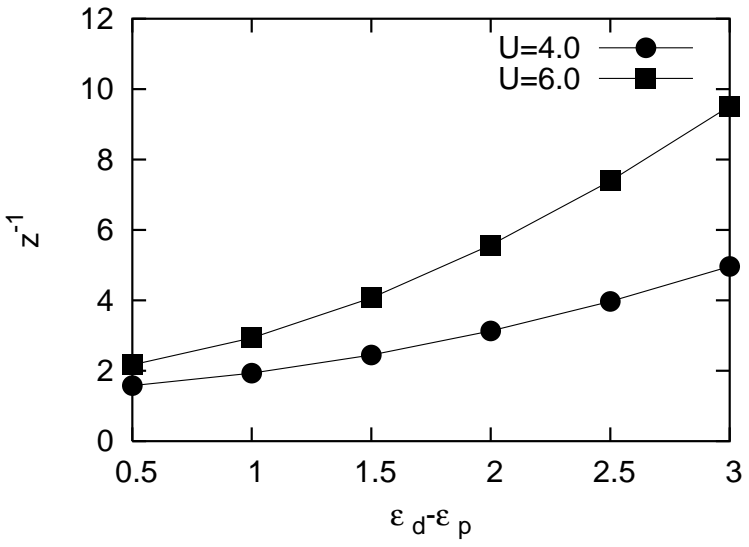

Fig. 8. $\varepsilon_{d}-\varepsilon_{p}$ dependence of $z^{-1}=\left\langle z^{-1}(\boldsymbol{k})\right\rangle$.

to say, $\varepsilon_{d}-\varepsilon_{p}$ is large for LSCO and small for YBCO, in this model. In Figs. 6 and 7, we show the $\varepsilon_{d}-\varepsilon_{p}$ dependences of $\rho_{d}(0)$ and $n_{d}$. When $\varepsilon_{d}-\varepsilon_{p}$ is large, these quantites also become large, and $n_{d}$ approaches unity. This indicates that LSCO is located in the relatively strongly correlated regime as compared with YBCO.

Now, let us evaluate the mass enhancement factor us- 


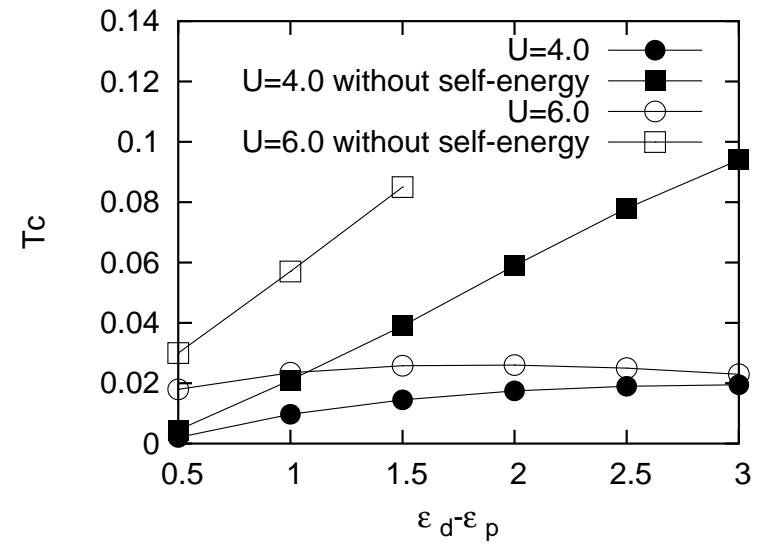

Fig. 9. $\varepsilon_{d}-\varepsilon_{p}$ dependence of $T_{\mathrm{c}}$.

ing the third-order perturbation theory.

$$
z^{-1}(\boldsymbol{k})=\left(1-\frac{\partial \Sigma(\boldsymbol{k}, \omega)}{\partial \omega}\right)_{\omega \rightarrow 0} .
$$

Fig. 8 shows the $\varepsilon_{d}-\varepsilon_{p}$ dependence of the average $z^{-1}=\left\langle z^{-1}(\boldsymbol{k})\right\rangle$. Here, $\langle\cdots\rangle$ represents the average over the momentum space. With increasing $\varepsilon_{d}-\varepsilon_{p}$, the mass enhancement factor increases. This is consistent with the finding that the mass enhancement of LSCO seems to be larger than that of YBCO, as mentioned above. ${ }^{7,8}$ This is due to the difference in $\rho_{d}(0)$. From another viewpoint, when the value of $\varepsilon_{d}-\varepsilon_{p}$ is large, $n_{d}$ approaches unity, and the electron mass is strongly enhanced by the strong electron correlation, similarly to the Mott transition. Such behavior has been markedly observed in the Ce-based heavy fermion systems.

Next, we discuss the superconducting transition temperature $T_{\mathrm{c}}$. From the experimental results, for large $\varepsilon_{d}-\varepsilon_{p}, T_{\mathrm{c}}$ is relatively low. In Fig. 9, we show the $\varepsilon_{d}-\varepsilon_{p}$ dependence of $T_{\mathrm{c}}$. $T_{\mathrm{c}}$ for $U=4.0$ increases as a function of $\varepsilon_{d}-\varepsilon_{p}$, and for $U=6.0$ is almost unchanged. Although the normal self-energy correction effect markedly suppresses $T_{\mathrm{c}}$, we cannot derive the results observed in the experiments within the present calculation. On the basis of the following reason, however, we can expect that by calculating the higher-order terms, we will obtain a better dependence of $T_{\mathrm{c}}$ on $\varepsilon_{d}-\varepsilon_{p}$. In Fig. 9, the filled (open) circles and squares denote $T_{\mathrm{c}}$ for $U=4.0$ $(U=6.0)$ with and without the normal self-energy correction, respectively. The difference is mainly due to the effect of the mass renormalization. The larger $\varepsilon_{d}-\varepsilon_{p}$ is, the more this effect increases. This tendency is considered to be more marked in the higher-order terms of the normal self-energy. On the other hand, higher-order terms of the pairing interaction were evaluated by Nomura et al. in the Hubbard model. The results show that the convergency for the pairing interaction is very good for the spin-singlet pairing near the half-filling. ${ }^{11}$ Namely, the contribution of the fourth-order terms to the pairing interaction is small compared with those of second-order and third-order terms. Here, we can expect the same trend. Thus, with the inclusion of the fourthorder terms in the Éliashberg equation, $T_{\mathrm{c}}$ will become relatively low for large $\varepsilon_{d}-\varepsilon_{p}$. This prediction is an important problem to be confirmed in the future.

In conclusion, we have investigated the mass enhancement factor and the superconducting transition temperature in the d-p model for the high- $T_{\mathrm{c}}$ cuprates. We have solved the Éliashberg equation using the third-order perturbation theory with respect to the on-site repulsion $U$. We find that when $\varepsilon_{d}-\varepsilon_{p}$ is large, the mass enhancement factor becomes large and $T_{\mathrm{c}}$ tends to be suppressed owing to the difference in $\rho_{d}(0)$. From another viewpoint, when the d-hole number approaches unity, the electron correlation between d-holes (electrons) becomes strong and the effective mass increases. In fact, LSCO with dhole number near unity shows strong mass enhancement . Here, we consider that $T_{\mathrm{c}}$ is given by the renormalization factor $z$ and $T_{\mathrm{c}}^{*}$ as

$$
T_{\mathrm{c}} \simeq z T_{\mathrm{c}}^{*},
$$

where $T_{\mathrm{c}}^{*}$ is the critical temperature determined by the calculation without any renormalization due to the normal self-energy correction. LSCO possesses small $z$ and exhibits low $T_{\mathrm{c}}$. Also, in the heavy fermion systems, when the number of f-electrons approaches unity, the effective mass is large and $T_{\mathrm{c}}$ is suppressed. This is the important unified theory which holds for all the strongly correlated electron systems from cuprates to heavy fermions. Thus, our calculation shows that, in order to systematically discuss the physical quantities such as $T_{\mathrm{c}}$ and the mass enhancement factor, we need to use the d-p model. The Hubbard Hamiltonian is insufficient to represent the difference among material systems.

\section{Acknowledgments}

Numerical calculation in this work was carried out at the Yukawa Institute Computer Facility.

1) P. A. Lee, N. Nagaosa and X-G. Wen: cond-mat/0410445.

2) T. Nakanishi, K. Yamaji and T. Yanagisawa: J. Phys. Soc. Jpn. 66 (1997) 294.

3) K. Kuroki and H. Aoki: Phys. Rev. B 56 (1997) 14287.

4) T. Moriya and K. Ueda: Adv. in Phys. 49 (2000) 555.

5) Y. Yanase, T. Jujo, T. Nomura, H. Ikeda, T. Hotta and K. Yamada: Phys. Rep. 387 (2003) 1.

6) G.-q. Zheng, Y. Kitaoka, K. Ishida and K. Asayama: J. Phys. Soc. Jpn. 64 (1995) 2524.

7) J. W. Loram, K. A. Mirza, J. R. Cooper and W. Y. Liang: Phys. Rev. Lett. 71 (1993) 1740.

8) N. Momono, T. Matsuzaki, M. Oda and M. Ido: J. Phys. Soc. Jpn. 71 (2002) 2832.

9) Y. Kitaoka, K. Ishida, S. Osugi, K. Fujiwara, G.-q. Zheng and K. Asayama: Appl. Magn. Reson. 3 (1992) 549.

10) Y. Husimi: Master thesis in Kyoto University.

11) T. Nomura and K. Yamada: J. Phys. Soc. Jpn. 72 (2003) 2053. 